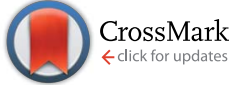

Cite this: RSC Adv., 2015, 5, 64471

Received 7th July 2015

Accepted 21st July 2015

DOI: 10.1039/c5ra13246k

www.rsc.org/advances

\section{Enhanced self-assembled monolayer treatment on polymeric gate dielectrics with ultraviolet/ozone assistance in organic thin film transistors $\uparrow$}

\begin{abstract}
Yan Yan, ${ }^{\mathrm{a}}$ Ye Zhou, ${ }^{\mathrm{a}}$ Long-Biao Huang, ${ }^{\mathrm{a}}$ Su-Ting Han, ${ }^{\mathrm{a}} \mathrm{Li}$ Zhou, ${ }^{\mathrm{a}}$ Zong-Xiang $\mathrm{Xu}{ }^{* c}$ and V. A. L. Roy *ab

Poly-4-vinylphenol (PVP), cross-linked PVP and poly(methyl methacrylate) (PMMA) are employed as polymeric insulators and chemical vapor deposition (CVD) is utilized to form self-assembled monolayers on the polymeric insulators. With a hexamethyldisilazane monolayer on polymeric dielectrics, an ordered molecular orientation is formed with larger grains resulting in improved carrier mobilities, and low threshold voltages $\left(V_{T}\right)$. Moreover, ultraviolet/ozone (UVO) treatment is used to enhance the alignment of HMDS monolayer on polymeric insulator surface and a time dependent effect is observed for UV/ozone treatment. For PVP and cross-linked PVP substrates, a short UVO exposure enhances the HMDS reaction on the polymer surface, and a long UVO exposure shows an adverse effect. On the other hand, PMMA is found to be more sensitive to UVO treatment and displayed performance degradation. These findings will be of value for solution processed insulators for printable electronic applications on flexible substrates.
\end{abstract}

\section{Introduction}

Over the past two decades, organic thin film transistors (OTFTs) have attracted wide scientific and technological interest because of their specific advantages of being inexpensive, lightweight and compatible with flexible substrates. ${ }^{1-7}$ In this aspect, solution-processed materials are attractive for organic electronics due to simple device fabrication processes such as spincoating, printing or drop-casting at low temperature under ambient conditions which are coupled with patterned printable techniques. ${ }^{8-10}$ Polymer dielectric materials exhibit great potential due to their complementary solubility and good insulating properties. ${ }^{11-14}$ The influences of a polymer dielectric material's structural properties on the growth of the semiconductor film and device electrical performance have been

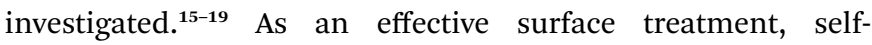
assembled monolayers (SAMs) are extensively applied on metals or oxides to modify the surface chemistry and have achieved obvious improvement in OTFT device performance. ${ }^{20-24}$ However, SAM treatment on polymer insulators and

${ }^{a}$ Department of Physics and Materials Science, City University of Hong Kong, Hong Kong SAR, China.E-mail: val.roy@cityu.edu.hk

${ }^{b}$ State Key Laboratory of Millimeter Waves, City University of Hong Kong, Hong Kong SAR, P. R. China

'Department of Chemistry, South University of Science and Technology of China, Shenzhen, P. R. China, 518055. E-mail: xu.zx@sustc.edu.cn

$\dagger$ Electronic supplementary information (ESI) available. See DOI: $10.1039 / \mathrm{c} 5 \mathrm{ra} 13246 \mathrm{k}$ the growth of semiconductors on such treated polymer insulators remain unclear. Therefore, it is necessary to study the interface between SAM-treated polymer insulators and semiconductor materials, as it determines the top molecular orientation and the final active film morphology, eventually influencing the device performance. Traditional dipping method may lead to polymer swelling and large roughness of the films, also the long reaction time in solvent introduces impurities at the polymer dielectric surface, which brings detrimental effect for device performance. ${ }^{25}$ In addition, compared with inorganic insulators, the reaction sites for polymer insulators are not sufficient, therefore added processing is needed to increase the number of reaction groups on the polymer surface. In order to avoid the polymer swelling and impurities due to organic solvent, chemical vapor deposition (CVD) was utilized to deposit hexamethyldisilazane (HMDS) monolayer on polymer insulators and ultraviolet/ozone (UVO) treatment was used to increase the reaction groups. ${ }^{26}$ Due to the sensitivity of polymer insulator surface to UVO treatment, the exposure effect and the respective device performance have been also analysed.

Here, we studied the effect of HMDS treatment on polymer dielectric surface for pentacene based OTFTs. Poly-4vinylphenol (PVP), cross-linked PVP (CPVP) and poly(methyl methacrylate) (PMMA) were employed as polymer insulators to study the effect of hydroxyl group density on the dielectric surface. OTFTs with diverse polymer insulators including PVP, cross-linked PVP (CPVP), PMMA and their HMDS-treated counterparts (H-PVP, H-CPVP, H-PMMA), finally UVO and 
HMDS-treated devices (UVO-H-PVP, UVO-H-CPVP and UVO-HPMMA) have been analysed. The thin film morphological change of polymer and pentacene were confirmed by the atomic force microscopy (AFM). The thin film structure and molecular orientation of pentacene on treated or non-treated surfaces were determined by X-ray diffraction (XRD). The changes of polymer dielectrics surface property were observed by X-ray photoemission spectroscope (XPS). The OTFT devices with HMDS treatment on polymer dielectrics exhibited better mobility, low threshold voltage as well as high on/off ratio $\left(10^{5}\right.$ to $\left.10^{6}\right)$. Moreover, in order to improve the SAM reaction with polymer insulators, the influence of UV/ozone exposure on the SAM treatment was elucidated, and the respective device characteristics were analyzed. For the PVP and CPVP insulator layer, a short UVO treatment obviously induced more $\mathrm{OH}$ groups leading to enhancement of the HMDS reaction on the polymer surface, and resulted in an ordered self-assembled molecular orientation, larger pentacene grain size and better device performance compared with no UVO exposure. While for long UVO exposure, the OTFTs performance showed a gradual degradation of performance. Obvious degeneration of polymer layer hence decrease in device performance was observed in PMMA samples due to its sensitivity to UVO exposure. The devices on diverse polymer dielectrics with HMDS treatment demonstrated improved electrical performance, indicating that SAM treatment is suitable and advantageous for printed electronics based on polymer insulators.

\section{Experimental}

\subsection{Materials}

Hexamethyldisilazane (HMDS), poly-4-vinylphenol (PVP, $M_{\mathrm{w}}=$ $\left.25000 \mathrm{~g} \mathrm{~mol}^{-1}\right)$, propylene glycol monomethyl ether acetate (PGMEA), poly(melamine-co-formaldehyde) (PMF), poly(methyl methacrylate) (PMMA, $M_{\mathrm{w}}=120000 \mathrm{~g} \mathrm{~mol}^{-1}$ ) and pentacene were purchased from Aldrich. All of the above chemicals and solvents were used without further purification.

\subsection{Polymeric dielectric film preparation}

PVP (100 $\mathrm{mg} \mathrm{ml}^{-1}$ ) and PMMA (50 $\mathrm{mg} \mathrm{ml}^{-1}$ ) were prepared in PGMEA and toluene solvent respectively. Cross-linked PVP (100 $\mathrm{mg} \mathrm{ml}^{-1}$ ) was prepared using a cross-linking agent PMF in a ratio of $2: 1$ by weight in PGMEA followed by an ultrasonication process to further enhance the solubility. Prior to spin-coating, solutions were filtered through a $0.2 \mu \mathrm{m}$ syringe filter. All the polymer insulators (PVP, cross-linked PVP and PMMA solutions) were spin-coated on heavily doped $\mathrm{n}^{++}$ Si surfaces with a speed of 2500 RPM for $1 \mathrm{~min}$. Finally, the crosslinked PVP samples were annealed in the vacuum oven at $200{ }^{\circ} \mathrm{C}$ for $2 \mathrm{~h}$ and PVP, PMMA substrates were annealed at $150^{\circ} \mathrm{C}$ for 1 $\mathrm{h}$ on a hot plate under a $\mathrm{N}_{2}$ atmosphere in a glove box.

\subsection{Polymeric dielectric surface modification}

The polymeric dielectric substrates were treated by UVO (Jelight Inc. 42-220 with a wavelength of $253.7 \mathrm{~nm}$ ) for different duration $(0,10 \mathrm{~s}, 30 \mathrm{~s}, 60 \mathrm{~s}, 300 \mathrm{~s})$, and then modified with HMDS molecules. CVD of HMDS was carried out in a desiccator under vacuum environment. Substrates were placed 3-5 $\mathrm{cm}$ above a glass dish containing $1 \mathrm{ml}$ HMDS solution. After 2 hours exposure under vacuum at room temperature $\left(20{ }^{\circ} \mathrm{C}\right)$, the substrates were cleaned to remove excessive self-assembled layer molecules. Then, substrates were placed on a hot plate at $150{ }^{\circ} \mathrm{C}$ for $30 \mathrm{~min}$ to form an ordered SAM arrangement in the Mbraun nitrogen glove box.

\subsection{Device fabrication and characterization}

Top-contact/bottom-gate transistors were fabricated on heavily doped silicon substrates coated with $400 \mathrm{~nm}$ PVP, $500 \mathrm{~nm}$ crosslinked PVP or $200 \mathrm{~nm}$ PMMA by spin-coating method. Pentacene was deposited on the dielectric insulator by thermal evaporation at rate of $0.2 \AA^{-1}$ and a thickness of $50 \mathrm{~nm}$. A gold film $(100 \mathrm{~nm})$ was vacuum sublimed on pentacene through a shadow mask $(L / W=30 \mu \mathrm{m} / 1000 \mu \mathrm{m})$ with a speed of $0.5 \AA \mathrm{s}^{-1}$ as source/drain electrodes. The configuration of pentacene based top-contact bottom-gate OFETs is shown in Fig. S1. $\dagger$ A Rame-hart Model 250-F1 Standard Goniometer with DROP image Advanced 2.1 was used to measure the static contact angle. A drop of deionized water was placed on polymer surface using a microsyringe at room temperature, and the contact angle at three different positions were measured to fit YoungLaplace curve around the drop. The surface morphologies of pentacene and HMDS-treated polymer surfaces were examined by the AFM (VEECO Multimode V, tapping mode). XRD patterns of semiconductor films on polymer insulators were recorded by a Rigaku Smartlab, collecting the diffraction data in a $2 \theta$ range of $3-30^{\circ}$ with a step-size of $0.02^{\circ}(2 \theta)$. X-ray photoelectron spectroscopy (XPS) was measured by Physical Electronics PHI 5802 with a monochromatic $\mathrm{Al} \mathrm{K} \alpha$ X-ray source. The device characterization was performed at room temperature in the MBraun nitrogen glove box by the semiconductor parameter analyzer (Agilent 4155C), Keithley 2612 source meter and HP 4284A LCR meter.

\section{Results and discussion}

\subsection{HMDS treatment without UVO}

Growth of pentacene film on dielectric layer is influenced by deposition condition as well as the chemical and physical properties of the surface. ${ }^{27,28}$ In order to avoid the effect of deposition conditions including deposition rate, substrate temperature and film thickness, all samples were prepared under same conditions $\left(0.2 \AA \mathrm{s}^{-1}\right.$, room temperature and a thickness of $50 \mathrm{~nm}$ ). The dielectric surface properties (surface roughness and surface energy) were characterized by AFM and contact angle measurements. The root-mean-square (RMS) roughness of the insulator layer was obtained on a $2 \mu \mathrm{m} \times 2 \mu \mathrm{m}$ area (Fig. S2 $\dagger$ ). Although the HMDS treatment slightly increases the substrate roughness, (all the surface roughness reached a value below $5 \AA$ ), it has negligible effect on the charge transport at the insulator/pentacene interface. ${ }^{29,30} \mathrm{X}$-ray photoemission spectroscope (XPS) was utilized to further investigate the interface characteristics of the HMDS layers. The $\mathrm{Si}_{2 \mathrm{p}}$ peaks 

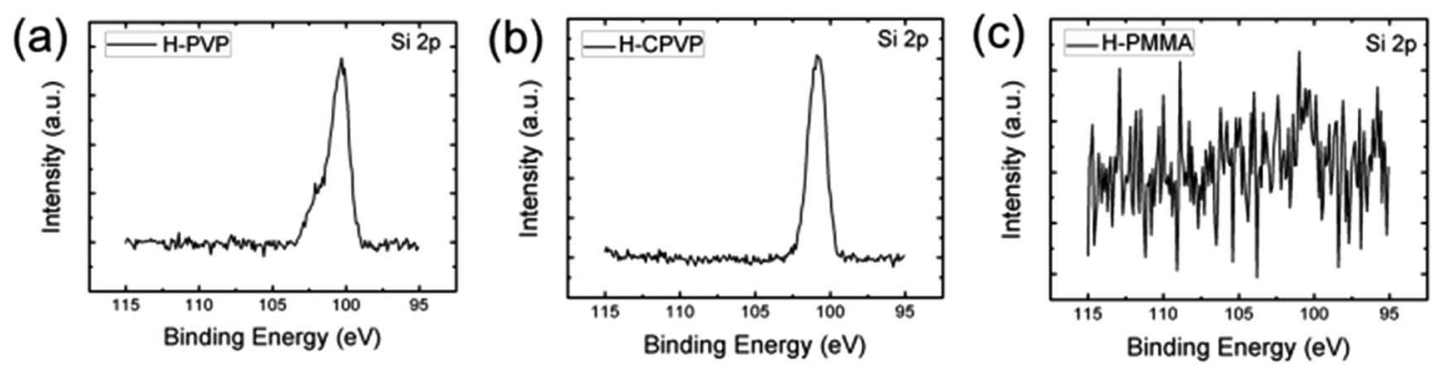

Fig. 1 XPS spectra of HMDS treatment polymer dielectric films (a) PVP (b) cross-linked PVP (c) PMMA with HMDS modification.

(a)

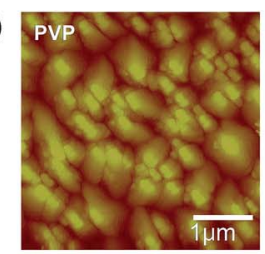

(d)

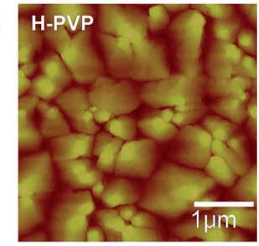

(b)

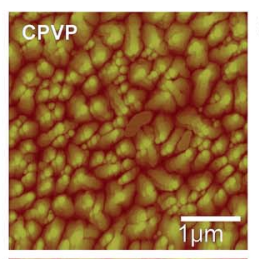

(e)

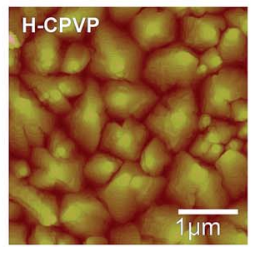

(c)

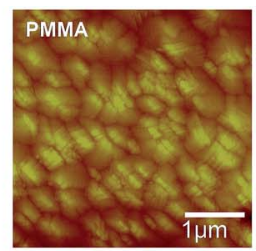

(f)

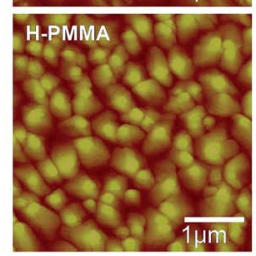

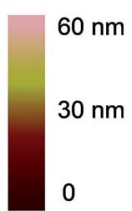

$\mathrm{nm}$

Fig. 2 Tapping mode AFM height image of $50 \mathrm{~nm}$-thick pentacene films grown on various dielectric layers: (a and d) PVP (b and e) cross-linked PVP, (c and f) PMMA, without or with HMDS modification.
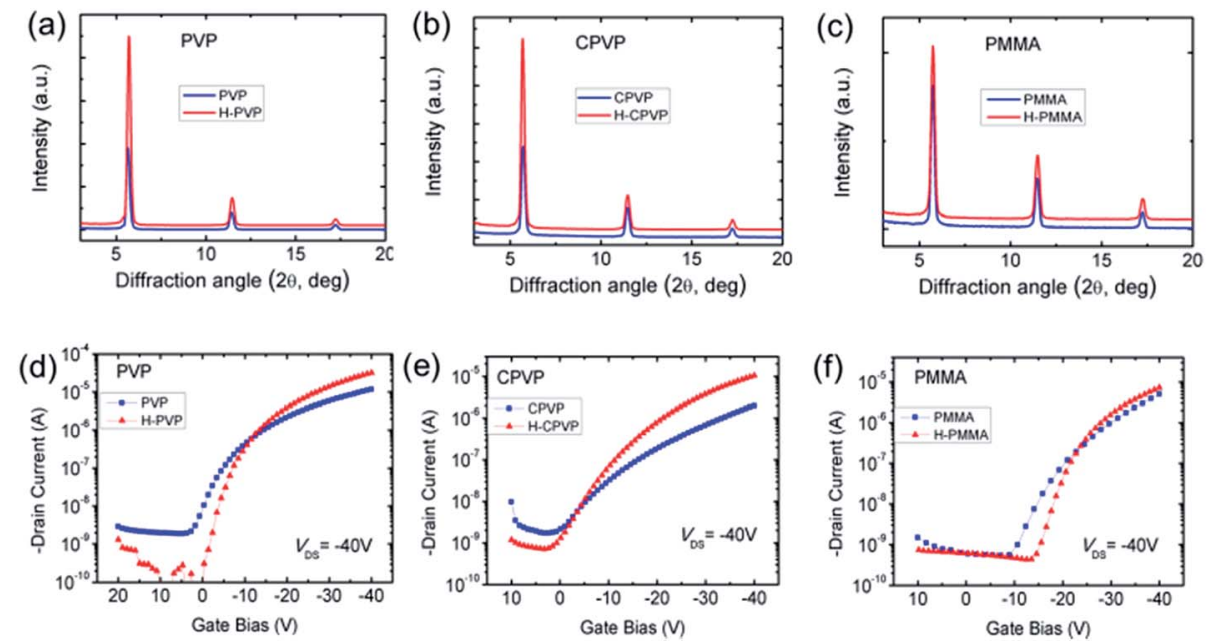

Fig. 3 XRD patterns and transfer characteristics of the OTFTs on various dielectric layers (a and d) PVP, (b and e) cross-linked PVP, and (c and f) PMMA without or with HMDS modification.

from the HMDS treated PVP and CPVP surface are shown in Fig. 1a and b respectively which proves successful modification of HMDS treated polymer surface. The $\mathrm{Si}_{2 \mathrm{p}}$ peak in CPVP surface shows that the reaction of hydroxyl group on the surface of cross-linker is not absolute. Some residual hydroxyl groups still exit on the surface of CPVP for the reaction with HMDS molecules. However, for the HMDS treated PMMA substrate, no peak is observed as shown in Fig. 1c, which demonstrates that without $\mathrm{OH}$ group on the PMMA surface, the HMDS treatment is not successful. The HMDS layer connects via simple physical adsorption can be totally removed in the cleaning process. The contact angle of various substrates is summarized in Table S1. $\dagger$ The near $75^{\circ}$ contact angle after HMDS treatment demonstrates that PVP, CPVP substrates were modified by methyl groups. Fig. 2a to $\mathrm{f}$ shows the TM-AFM topographies of $50 \mathrm{~nm}$ thick pentacene films on PVP ( $a$ and d), CPVP (b and e) and PMMA 
(c and f) polymer surfaces with and without HMDS treatment on a $4 \mu \mathrm{m} \times 4 \mu \mathrm{m}$ area. Significant morphological changes are found after HMDS treatment for the film on PVP and CPVP, the pentacene grain size increases obviously from 0.42 to $0.70 \mu \mathrm{m}$ and 0.26 to $0.60 \mu \mathrm{m}$ respectively. As shown in Fig. 3a to c, the presence of (001) reflection of pentacene thin film which is referred as "thin film phase" is observed, indicating that pentacene molecules in the film structure is oriented parallel to the insulator surfaces with (001) planes $\left(d_{(001)}=15.1-15.4 \AA\right)$. Additionally, the higher peak intensity is achieved due to the larger pentacene grains and higher crystallinity from the enhanced molecular orientation of pentacene monolayers influenced by the SAM treatment, which shows consistency with AFM images. The pentacene molecules grow as faceted islands on the interface with HMDS treatment and form single crystallike morphology of pentacene films. In each island, during the crystal growing process, there would hardly be any internal defects, which is advantageous for charge transport. ${ }^{31}$ Fig. $3 \mathrm{~d}-\mathrm{f}$ shows the transfer characteristics of OTFTs. From the Fig. $3 \mathrm{~d}-\mathrm{f}$ and Table $\mathrm{S} 1, \dagger$ a reduced off-current, obviously increased mobility and positive-shift threshold voltage $\left(V_{\mathrm{T}}\right)$ due to HMDS treatment for various polymer insulators could be found in H-PVP and H-CPVP devices. From these results, HMDS treatment found to reduce the number of trapping states contributing to off current suppression and improve charge transport properties of the device. ${ }^{32,33}$ Furthermore, the pentacene film based on HMDS treatment exhibited better crystallinity and larger grain size which lead to the enhancement of device performance. According to the above results, the "thin film phase" plays a key role in better device performance. At the same time, among the three kinds of polymer insulators after HMDS modification, PVP shows the highest enhancement in pentacene film quality and corresponding device performance, followed by CPVP while almost no improvement is observed for the devices with PMMA as insulator. This is due to various hydroxyl group densities on the dielectric surface. The amount of hydroxyl groups on the surface of PVP is more than that of CPVP surface due to the reaction of hydroxyl group with the cross-linker in the case of CPVP whereas PMMA surface has no hydroxyl group.

\subsection{The effect of UVO exposure}

HMDS is a self-assembled monolayer (SAM) with methyl chains to modify the surface energy of gate insulators and decreases the traps induced by $\mathrm{OH}$ groups on gate insulators. The CVD processed HMDS treatment avoids the influence of impurities and swelling effect due to long immersing time of polymer samples in organic solvents. ${ }^{25}$ However, SAM treatment is a process to create ordered molecular arrangement through the adsorption of an active surfactant on the top surface, the ordering of SAMs is influenced by adhesiveness of the surface and intermolecular interactions between the HMDS. ${ }^{34}$ Due to insufficient $\mathrm{OH}$ groups on the polymer surface, the formation of SAM layer might not be as ideal as on the surface of inorganic insulator such as $\mathrm{SiO}_{2}$. Hence further processing is essential to modify the polymer insulator surface. UVO is a simple, inexpensive and well established method for removing organic and other contaminants from the surface. Related works has been reported to utilize UVO treatment to change the chemical structure of inorganic or polymer insulators in order to improve the electrical performance. ${ }^{35-37}$ Here, we study the influence of UVO and its reaction with the polymer surface. In order to create more $\mathrm{OH}$ groups on the polymer insulator surface, prior to HMDS surface modification UVO treatment has been employed with various time duration $(0,10 \mathrm{~s}, 30 \mathrm{~s}, 60 \mathrm{~s}, 300 \mathrm{~s})$. Fig. S3† shows the contact angle change with UV/ozone exposure time for PMMA sample. We can find that the contact angle reduces from $83^{\circ}$ to $60^{\circ}$ after $10 \mathrm{~s}$ exposure and finally come to $35^{\circ}$ after $300 \mathrm{~s}$ exposure. Meanwhile, after the HMDS treatment on the UV/ozone treated PMMA substrate, the contact angle comes back to around $75^{\circ}$, indicating that the HMDS layer is well coated on the top. The TM-AFM image of $50 \mathrm{~nm}$-thick pentacene films on PVP (a and d), CPVP (b and e) and PMMA (c and f) on HMDS-treated polymer substrates for a $4 \mu \mathrm{m} \times 4 \mu \mathrm{m}$ area are shown in Fig. 4. The difference is the duration of UVO exposure prior to HMDS treatment. For UVO-H-PVP and UVO-H-CPVP samples, the growth of pentacene film exhibited larger grain size than the ones treated for longer time. Additionally, in comparison with samples of H-PVP and H-CPVP (Fig. 2), even larger grains are observed for samples UVO-H-PVP and UVO-H-CPVP, which implies that short duration UVO treatment induced more $\mathrm{OH}$ groups and enhanced the HMDS reaction on PVP and CPVP surface. For UVO-H-PMMA substrates, after $10 \mathrm{~s}$ UVO exposure, no obvious change in film morphology is found while samples with $300 \mathrm{~s}$ exposure shows a dramatic reduction in grain size. In order to understand the influence of long duration UVO exposure on surface roughness, RMS of treated polymer dielectric surfaces such as UVO-H-PVP, UVO-H-CPVP and UVO-H-PMMA with 300 s UVO exposure was measured (Table S2 and Fig. $\mathrm{S} 4 \dagger$ ) and no obvious change is found. Fig. 5a-c shows the XRD pattern of UVO influence on HMDS-treated pentacene films. The UVO-H-PVP and UVO-H-CPVP samples with $10 \mathrm{~s}$ UVO exposure found to exhibit enhanced crystallinity compared to samples without UVO treatment. Meanwhile for the $300 \mathrm{~s}$ UVOtreated samples, the peak intensity reduced dramatically and found to be even lower than the samples with no combined treatment (UVO and HMDS). While for the PMMA insulator, the intensity exhibited a continuous downward trend due to UVO treatment. At the same time, "bulk phase" is observed when the UVO exposure reached 300 s. A "bulk phase" $\left(d_{(001)}=14.1 \AA\right)$ starts to appear when the pentacene film becomes thicker or the deposition temperature is changed and from the perspective of energetics, the "bulk phase" structure is more stable than "thin film phase" structure. ${ }^{38-40}$ The coexistence of two crystalline phases means there are two different interlayer spacing and layer orientation with respect to the substrate in pentacene film. These mixed crystalline phase structure might contribute large resistance for the charge transport in pentacene film. The change in the grain size and carrier mobility is shown in Fig. $5 \mathrm{~d}$ to $\mathrm{f}$, which shows a similar trend. The data display in the left part is for the device without any treatment (UVO and HMDS). For the UVO-H-PVP and UVO-H-CPVP samples with $10 \mathrm{~s}$ UVO exposure, the mobility achieves the largest value of 0.45 and 
(a)

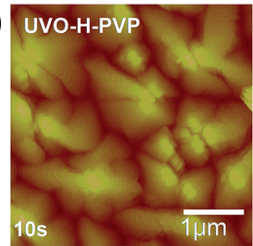

(d)

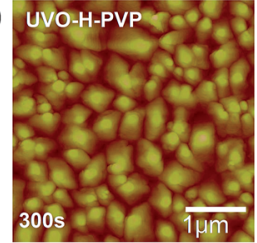

(b)

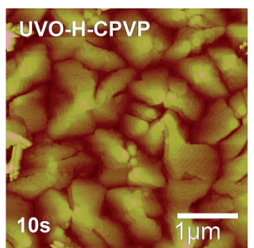

(e)

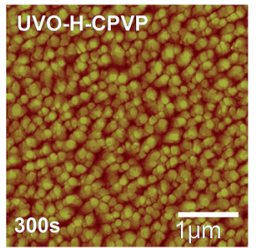

(c)

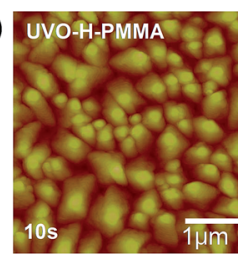

(f)

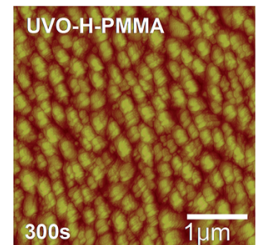

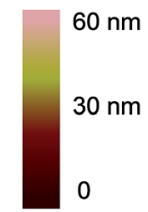

$0 \mathrm{~nm}$

Fig. 4 Tapping mode AFM height image of $50 \mathrm{~nm}$-thick pentacene films grown on various dielectric layers with UVO treatment (10 s and $300 \mathrm{~s}$ ): ( $a$ and $d$ ) PVP ( $b$ and e) cross-linked PVP, (c and f) PMMA, all the samples are modified with HMDS after UVO treatment.
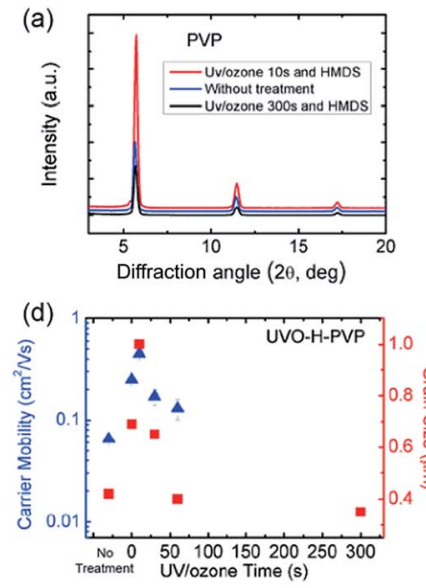
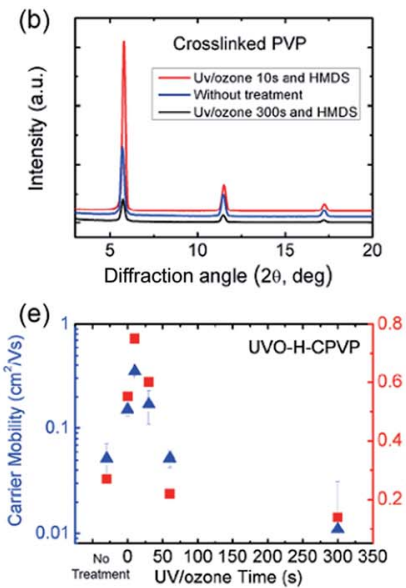
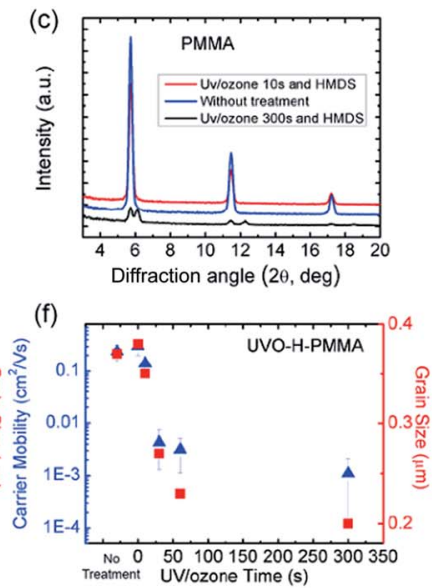

Fig. 5 XRD patterns of $50 \mathrm{~nm}$-thick pentacene film grown on various UVO treated polymer insulators: (a) PVP, (b) cross-linked PVP, and (c) PMMA. Carrier mobility and grain size change dependence on UVO exposure time: (d) PVP, (e) cross-linked PVP, and (f) PMMA.

$0.34 \mathrm{~cm}^{2} \mathrm{~V}^{-1} \mathrm{~s}^{-1}$ respectively and reduces if further UVO exposure is carried out. In particular, after $300 \mathrm{~s}$ of UVO treatment the OTFTs with UVO-H-PVP resulted in large leakage currents and exhibited no field-effect (Fig. S5 $\dagger$ ) behaviour. For PMMA samples, UVO showed a negative effect on device electrical performance. The UVO treatment of a polymer could result in complex changes on the chemical structure of polymer surface. Here, the different polymers showed distinct sensitivity for the UVO exposure. Koo et al. studied the effect of UVO treatment on the hysteresis of pentacene thin film transistors with polymer gate insulator, and an enlarged hysteresis was observed due to the increase of $\mathrm{OH}$ group which was confirmed by FT-IR spectra. ${ }^{35}$ Hysteresis measurement for OFETs with various polymeric dielectrics and treatments have also been performed and shown in Fig. S6 and S7, $\dagger$ showing a regular trend with surface properties change. Here, we utilized XPS to analyze $\mathrm{OH}$ groups after UVO treatment. As shown in Fig. S8, $\dagger$ the UVO-H-PMMA substrate shows an obvious $\mathrm{Si}_{2 \mathrm{p}}$ peak which proves that the HMDS molecules reacted successfully on the UVO treated PMMA dielectric layer surface. In comparison with Fig. $1 c$ in which no $\mathrm{Si}_{2 \mathrm{p}}$ peak is observed, the appearance of $\mathrm{Si}_{2 \mathrm{p}}$ peak as shown in Fig. $\mathrm{S}^{\dagger} \uparrow$ demonstrates that the UVO play an important role in generating hydroxyl group on PMMA surface. Lim et al. also reported the effect of hydroxyl group influence on the device electrical reliability of OTFTs fabricated using PVP film as insulator and pentacene film as active layer. ${ }^{41}$ We believe that UVO process impacts various polymer surfaces diversely with respect to exposure time. In general, UV irradiation removes the organic contaminants and increases the number of $\mathrm{OH}$ groups if the exposure duration is short, which is beneficial for the modification of methyl chains on the polymer surface. The good HMDS arrangement increases the pentacene grain size on the top as shown in Fig. 4a-c, contributing to the much higher mobilities in device as shown in Fig. 5. However, if the exposure time is long, the UVO treatment degrades the polymer chain, generally originating from the scission of C-C bonds. ${ }^{42}$ Due to chain scission, photolysis and/or dissociation of side group, the chemical structures of polymer insulator surface become inhomogeneous, showing detrimental effects on selfassembled monolayers arrangement. Meanwhile, the ozone from the equipment enhances the effect of surface inhomogeneous property. Fig. S9† shows the surface morphology change of 


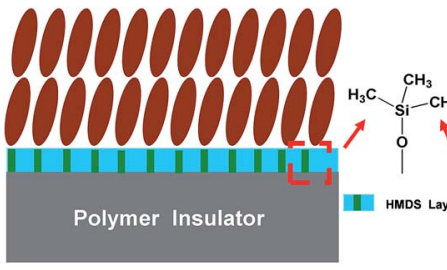

(a) Short UVO Treatment

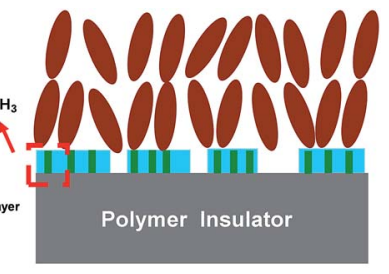

(b) Long UVO Treatment
Fig. 6 Schematic diagram of pentacene molecules grown on substrates with short (a) and long (b) UVO treatment and HMDS arrangement.

HMDS monolayers on $10 \mathrm{~s}$ and $300 \mathrm{~s}$ UVO treated PVP polymeric surface. An obvious RMS increase of top HMDS layer is observed from $2.6 \AA$ to $9.3 \AA$ due to worse monolayers arrangement. The rough polymer interface and poor SAM arrangement is harmful to the pentacene film growth on the top as shown in Fig. 4d-f. The group size of pentacene molecular show an obvious decrease for all PVP, CPVP and PMMA based devices and the mobilities reduce more than one order of magnitude as shown in Fig. 5.

Fig. 6 schematic explains the UVO exposure influence on the performance of OTFTs based on polymer insulator with HMDS treatment. The pentacene molecular orientation is affected by the HMDS arrangement on the polymer insulator surface. For the short UVO treatment, a uniform OH group layer is generated, leading to the formation of relatively ordered SAM arrangement after HMDS treatment. The ordered SAM layer induces better pentacene orientation and larger grain size, achieving high electrical performance. On the contrary, due to long time UV/exposure, inhomogeneous surface chemical properties containing various bonds are formed which obstructs the uniform SAM reaction, resulting in decreased electrical performance. Cho and co-worker controlled the alkyl chains alignment with SAM by changing the preparation temperature, and an obvious coexistence of two crystalline phases were observed in XRD measurements, demonstrating that SAM arrangement plays a critical role in determining OTFTs performance. ${ }^{39}$ The result confirms our supposition of UVO influence on SAM alignment in polymer surface. The current stability including bias stress effect and air stability have also been measured and shown in Fig. S10 and S11. $\dagger$

\section{Conclusions}

In summary, we investigated SAM treatment on PVP, crosslinked PVP and PMMA insulators and respective pentacene based OTFT devices. In addition, UVO exposure influence on self-assembled monolayer alignment was analyzed. The formation of SAM by CVD is found to be a suitable treatment method on polymer surface. The UVO treatment manipulates the polymer insulator surface properties, and exhibited distinct sensitivity. Appropriate UVO exposure on polymer insulator induces uniform HMDS arrangement, resulting in better pentacene morphology and electrical performance. Additionally, as the sensitivities to UVO treatment are distinct for various polymers, depending on specific polymer insulator, the UVO treatment conditions should be selectively controlled. These results demonstrate a guideline for optimizing OTFTs performance with solution-processed polymer insulator, which is beneficial for printed electronic devices based on flexible substrates.

\section{Acknowledgements}

We acknowledge grants from City University of Hong Kong's Project no. 7004198, the Research Grants Council of the Hong Kong Special Administrative Region (Project No. T23-713/11) and Shenzhen overseas high level talents innovation plan of technical innovation project (Project No. KQCX20130628152708144).

\section{Notes and references}

1 H. Sirringhaus, Adv. Mater., 2005, 17, 2411.

2 L. A. Majewski, R. Schroeder and M. Grell, Adv. Mater., 2005, 17, 192.

3 S. Liu, W. M. Wang, A. L. Briseno, S. C. B. Mannsfeld and Z. Bao, Adv. Mater., 2009, 21, 1217.

4 Y. Wen, Y. Liu, Y. Guo, G. Yu and W. Hu, Chem. Rev., 2011, 111, 3358.

5 Y. Guo, G. Yu and Y. Liu, Adv. Mater., 2010, 22, 4427.

6 C.-a. Di, F. Zhang and D. Zhu, Adv. Mater., 2013, 25, 313.

7 D. Braga and G. Horowitz, Adv. Mater., 2009, 21, 1473.

8 L. Zhang, C.-a. Di, G. Yu and Y. Liu, J. Mater. Chem., 2010, 20, 7059.

9 J. Smith, R. Hamilton, I. McCulloch, N. Stingelin-Stutzmann, M. Heeney, D. D. C. Bradley and T. D. Anthopoulos, J. Mater. Chem., 2010, 20, 2562.

10 H. E. Katz, Chem. Mater., 2004, 16, 4748.

11 Y. Zhou, S.-T. Han, Z.-X. Xu and V. A. L. Roy, J. Mater. Chem., 2012, 22, 4060.

12 T. B. Singh and N. S. Sariciftci, Annu. Rev. Mater. Res., 2006, 36, 199.

13 M. E. Roberts, N. r. Queraltó, S. C. B. Mannsfeld, B. N. Reinecke, W. Knoll and Z. Bao, Chem. Mater., 2009, 21, 2292.

14 A. Facchetti, M. H. Yoon and T. J. Marks, Adv. Mater., 2005, 17, 1705.

15 X.-H. Zhang, S. P. Tiwari and B. Kippelen, Org. Electron., 2009, 10, 1133.

16 W. Xu and S.-W. Rhee, Org. Electron., 2011, 12, 2040.

17 S. Pyo, J. Choi, Y. Oh, H. Son and M. H. Yi, Appl. Phys. Lett., 2006, 88, 173501.

18 Y. Oh, S. Pyo, M. H. Yi and S.-K. Kwon, Org. Electron., 2006, 7, 77.

19 A. L. Deman, M. Erouel, D. Lallemand, M. Phaner-Goutorbe, P. Lang and J. Tardy, J. Non-Cryst. Solids, 2008, 354, 1598.

20 J. Youn, G. R. Dholakia, H. Huang, J. W. Hennek, A. Facchetti and T. J. Marks, Adv. Funct. Mater., 2012, 22, 1856.

21 S. D. Wang, T. Miyadera, T. Minari, Y. Aoyagi and K. Tsukagoshi, Appl. Phys. Lett., 2008, 93, 043311.

22 W. Shao, H. Dong, L. Jiang and W. Hu, Chem. Sci., 2011, 2, 590. 
23 D. Knipp, R. A. Street, A. Volkel and J. Ho, J. Appl. Phys., 2003, 93, 347.

24 S. A. DiBenedetto, A. Facchetti, M. A. Ratner and T. J. Marks, Adv. Mater., 2009, 21, 1407.

25 S. Mototani, S. Ochiai, X. Wang, K. Kojima, A. Ohashi and T. Mizutani, Jpn. J. Appl. Phys., 2008, 47, 496.

26 J. Dong, A. Wang, K. Y. S. Ng and G. Mao, Thin Solid Films, 2006, 515, 2116.

27 H. Yanagisawa, T. Tamaki, M. Nakamura and K. Kudo, Thin Solid Films, 2004, 398, 464.

28 A. C. Mayer, R. Ruiz, R. L. Headrick, A. Kazimirov and G. G. Malliaras, Org. Electron., 2004, 5, 257.

29 S. Steudel, S. D. Vusser, S. D. Jonge, D. Janssen, S. Verlaak, J. Genoe and P. Heremans, Appl. Phys. Lett., 2004, 85, 4400.

30 S. E. Fritz, T. W. Kelley and C. D. Frisbie, J. Phys. Chem. B, 2005, 109, 10574.

31 H. Yang, T. J. Shin, M.-M. Ling, K. Cho, C. Y. Ryu and Z. Bao, J. Am. Chem. Soc., 2005, 127, 11542.

32 I. Yagi, K. Tsukagoshi and Y. Aoyagi, Appl. Phys. Lett., 2005, 86, 103502.
33 T. Miyadera, S. D. Wang, T. Minari, K. Tsukagoshi and Y. Aoyagi, Appl. Phys. Lett., 2008, 93, 033304.

34 A. Ulman, Chem. Rev., 1996, 96, 1533.

35 J. B. Koo, S. Y. Kang, I. K. You and K. S. Suh, Solid-State Electron., 2009, 53, 621.

36 S. J. Han, J.-H. Kim, J. W. Kim, C.-K. Min, S.-H. Hong, D.-H. Kim, K.-H. Baek, G.-H. Kim, L.-M. Do and Y. Park, J. Appl. Phys., 2008, 104, 013715.

37 D. Guo, S. Entani, S. Ikeda and K. Saiki, Chem. Phys. Lett., 2006, 429, 124.

38 H. Yang, S. H. Kim, L. Yang, S. Y. Yang and C. E. Park, Adv. Mater., 2007, 19, 2868.

39 H. S. Lee, D. H. Kim, J. H. Cho, M. Hwang, Y. Jang and K. Cho, J. Am. Chem. Soc., 2008, 130, 10556.

40 C. D. Dimitrakopoulos, A. R. Brown and A. Pomp, J. Appl. Phys., 1996, 80, 2501.

41 S. C. Lim, S. H. Kim, J. B. Koo, J. H. Lee, C. H. Ku, Y. S. Yang and T. Zyung, Appl. Phys. Lett., 2007, 90, 173512.

42 D. Cheneler and J. Bowen, Soft Matter, 2013, 9, 344. 